\title{
Phenformin Hydrochloride
}

National Cancer Institute

\section{Source}

National Cancer Institute. Phenformin Hydrochloride. NCI Thesaurus. Code C81696.

The hydrochloride salt form of phenformin, an agent belong ing to the biguanide class of antidiabetics with antihyperglycemic activity. Phenformin is not used clinically due to the high risk of lactic acidosis that is associated with its use. 